




\title{
Pressure Rise in Run6 and Related Issues
}

\author{
S.Y. Zhang, H.C. Hseuh, C. Montag, V. Ptitsyn, D. Trbojevic
}

\section{Abstract}

The dynamic pressure rises in Run6 at unchanged straight sections are lower than that in Run5. Meanwhile in Run6, the beam is injected into the RHIC with slightly higher energy above the transition, which is suspected to reduce the electron cloud. If this effect is confirmed, then it might be used as another mitigation to the RHIC electron cloud for proton operations.

\section{Introduction}

For same vacuum chambers and same beam conditions, the dynamic pressure rises in Run5 were very similar to that in Run4. For same chamber and similar beam conditions, the pressure rises in Run6 are consistently lower than that in Run5. The enlarged difference of the injection energy above the transition energy, which is implemented in Run6, is suspected to play a role.

If confirmed, then this may explain a long lasting puzzle about the RHIC electron cloud, which has much larger bunch spacing than other machines with electron cloud problem. One of these machines is CERN SPS, which would have no problem if running with the RHIC bunch spacing of $108 \mathrm{~ns}$ with $2 \times 10^{11}$ protons per bunch. Probably not incidently, difference of the injection energy above the transition at SPS is much larger than RHIC.

It might be worth a beam study - toward a more fundamental mitigation for the RHIC electron cloud for proton operations.

\section{Pressure rise-Run6/Run5 vs. Run5/Run4}

In RHIC Run5 proton operations, it was noticed that the dynamic pressure rises at unchanged straight sections are very similar to Run4, under compa- 
rable beam conditions [1].

Figure 1 shows Blue beams of Fill 5350 in Run4 and Fill 7333 in Run5, both are in 110-bunch mode (bunch spacing of $108 \mathrm{~ns}$ ) and with $2 \times 10^{11}$ protons per bunch, and the pressure rises of 6 unchanged or little changed Blue Q3-Q4 straight sections are very similar. Table 1 shows peak pressure rises and the NEG installations at these Blue straight sections.

\begin{tabular}{c|c|c|c} 
Location & Peak Pressure & Peak Pressure & NEG Pipes \\
\hline & 5350 (Run4) & 7333 (Run5) & 2004,2005 \\
\hline \hline Bi1 & 6.6 & 3.2 & 0,0 \\
\hline Bo3 & 3.7 & 5.7 & 0,0 \\
\hline Bi4 & 7.1 & 4.7 & 0,0 \\
\hline Bo6 & 3.6 & 4.2 & 0,0 \\
\hline Bo10 & 15.6 & 3.9 & 0,3 \\
\hline Bi12 & 12.5 & 4.1 & 0,0 \\
\hline & $10^{-8}$ Torr & $10^{-8}$ Torr & Meters
\end{tabular}

Table 1: Peak pressure rise at pw3.2 of 6 unchanged or little changed Blue Q3-Q4 straight sections of Fill 5350 in Run4 and Fill 7333 in Run5, and the NEG pipe installations. The pressure rise at Bi12 is more variable due to the polarimeter there.

Figure 2 shows the Yellow beams of Fill 5350 in Run4 and Fill 7329 in Run5, both are in 110-bunch mode and with $1.5 \times 10^{11}$ protons per bunch, and the pressure rises of 6 unchanged or little changed Yellow Q3-Q4 straight sections are very similar. Table 2 shows peak pressure rises and the NEG installations at these Yellow straight sections. 

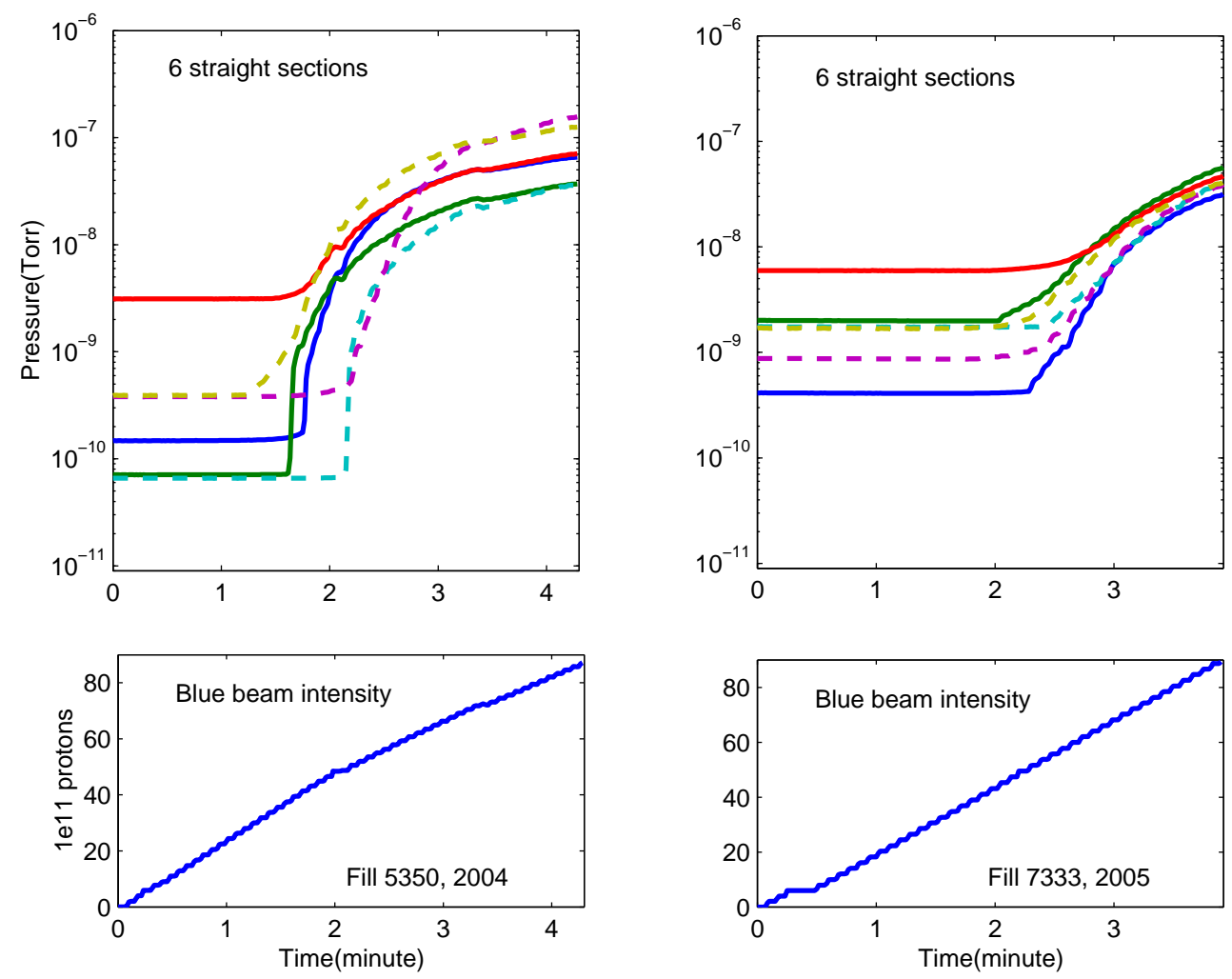

Figure 1: Pressure rises of Fill 5350 in Run4 and Fill 7333 in Run5. Both are Blue beams in 110-bunch mode (bunch spacing of $108 \mathrm{~ns}$ ) and with $2 \times 10^{11}$ protons per bunch. The pressure rises at pw3.2, located at the middle of the section, of 6 unchanged or little changed Blue Q3-Q4 straight sections are shown. Since the early injected bunches in 5350 were with higher bunch intensities, the pressure rise started earlier in the filling than that in 7333 . The pressure levels prior to the beam injection in 7333 were high, which were mostly the remnant from the beam injections occurred prior to this fill. 



Figure 2: Pressure rises of Fill 5350 in Run4 and Fill 7329 in Run5. Both Yellow beams are in 110 -bunch mode and with $1.5 \times 10^{11}$ protons per bunch. The pressure rises at pw3.2 of 6 unchanged or little changed Yellow Q3-Q4 straight sections are compared. Since the early injected bunches in 5350 were with higher bunch intensities, the pressure rise started earlier in the filling than that in 7329 . 


\begin{tabular}{c|c|c|c} 
Location & Peak Pressure & Peak Pressure & NEG Pipes \\
\hline \hline & 5350 (Run4) & 7329 (Run5) & 2004,2005 \\
\hline Yi3 & 0.15 & 0.48 & 0,0 \\
\hline Yo4 & 8.3 & 18.6 & 0,7 \\
\hline Yi7 & 38.2 & 27.8 & 0,0 \\
\hline Yo9 & 2.0 & 3.6 & 0,0 \\
\hline Yi11 & 0.55 & 0.9 & 0,0 \\
\hline Yo12 & 13.6 & 33.0 & 0,0 \\
\hline & $10^{-8}$ Torr & $10^{-8}$ Torr & Meters
\end{tabular}

Table 2: Peak pressure rise at pw3.2 of 6 unchanged or little changed Yellow Q3-Q4 straight sections of Fill 5350, 2004 and Fill 7329, 2005, and the NEG pipe installations. The vacuum pump at Yo4 pw3.2 was off for 7329 . The pressure rise at Yo12 is more variable due to the polarimeter there.

The pressure rise observed in Run6 is quite different from this scenario. For same straight sections, pressure rise in Run6 is consistently lower than that in Run5, under similar beam conditions. In Figure 3, the pressure rises at pw3.2 for 4 unchanged Blue and 5 unchanged Yellow Q3-Q4 straight sections are shown for Fill 7332 in Run5, and Fills 7554 and 7657 in Run6.

The beam conditions are compared in Table 3 .

\begin{tabular}{c|c|cc|c} 
Beam condition & 7332 (Run5) & 7554 (Run6) & 7657 (Run6) & \\
\hline \hline Bunch spacing & 108 & 108 & 108 & $n s$ \\
\hline Blue beam intensity & 140 & 157 & 137 & $10^{11}$ \\
\hline Yellow beam intensity & 135 & 144 & 131 & $10^{11}$ \\
\hline Blue bunch length & 7.9 & 7.3 & 5.7 & $n s$ \\
\hline Yellow bunch length & 7.8 & 7.4 & 5.9 & $n s$
\end{tabular}

Table 3: Beam condition of 7332 in Run5 and 7554, 7657 in Run6. Note that the 7554 has higher intensity and shorter bunches than 7332 , hence it is a stronger beam w.r.t. electron multipacting. Beam intensities in 7657 are a little lower than 7332 , but the bunches are shorter.

With the same bunch spacing, the beam intensity of 7554 in Run6 is higher, and the bunch length is smaller, than that of 7332 in Run5. Beam intensities in 7657 are a little lower than 7332 , but the bunches are shorter. Despite that, the dynamic pressure rises at all unchanged straight sections 

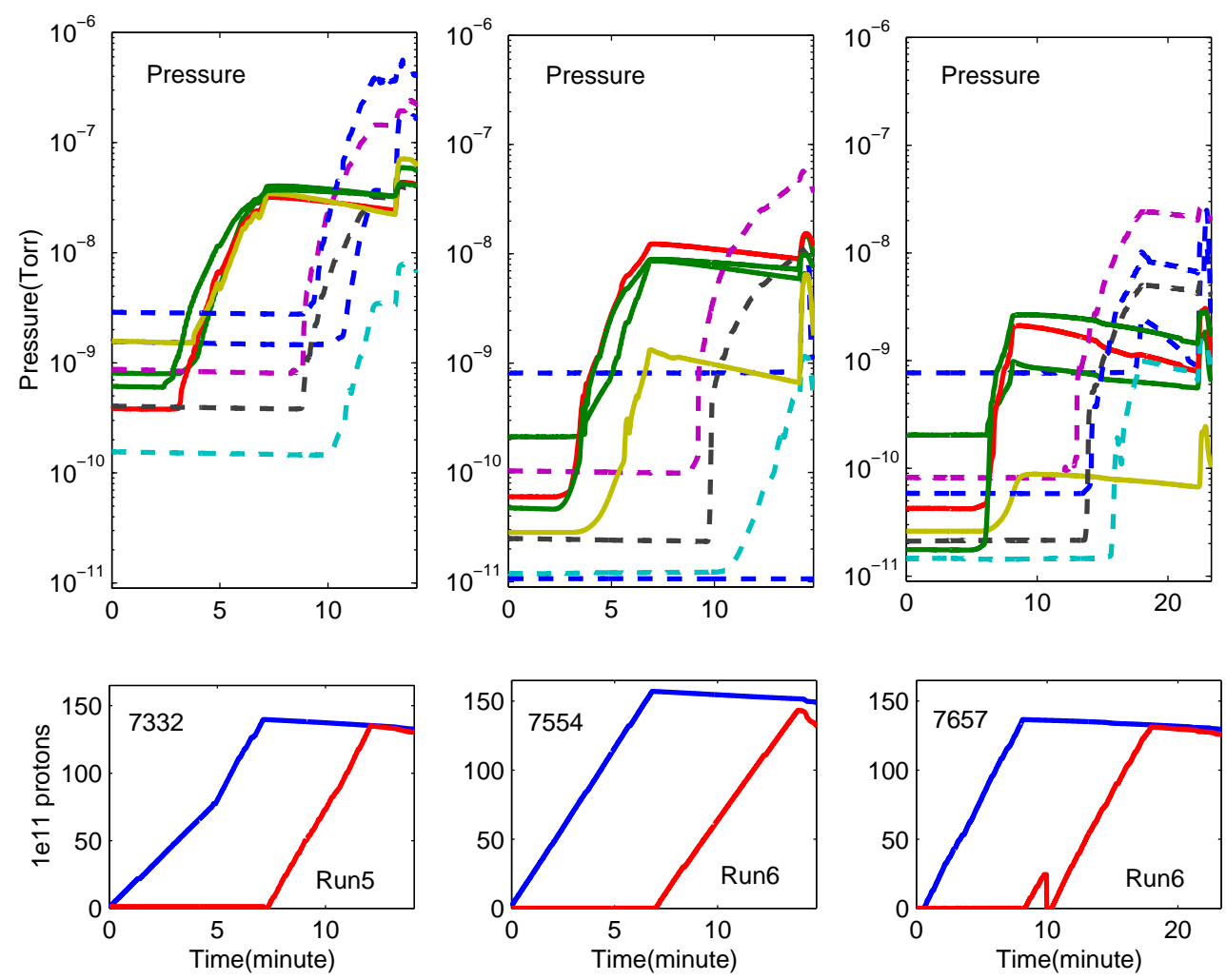

Figure 3: Pressure rises of Fill 7332 in Run5 and Fills 7554 and 7657 in Run6. All beams are in 110-bunch mode and with $\sim 1.3 \times 10^{11}$ protons per bunch. The pressure rises at pw3.2 of 4 unchanged Blue and 5 unchanged Yellow Q3-Q4 straight sections are compared. Solid lines for Blue, and dotted lines for Yellow. The pressure levels prior to the beam injection in 7332 in Run5 were high, which are mostly the remnant from the beam injections occurred prior to this fill. 
in both 7554 and 7657 are lower than that of 7332, without an exception. Table 4 shows peak pressure rises and the NEG installations at these straight sections.

\begin{tabular}{c|c|cc|c} 
Location & Peak Pressure & Peak Pressure & Peak Pressure & NEG Pipes \\
\hline & 7332 (Run5) & 7554 (Run6) & 7657 (Run6) & 2005,2006 \\
\hline \hline Bi1 & 4.4 & 1.5 & 0.3 & 0,0 \\
\hline Bo6 & 7.1 & 0.7 & 0.02 & 0,0 \\
\hline Bi8 & 4.2 & 1.0 & 0.3 & 12,12 \\
\hline Bo10 & 5.9 & 1.5 & 0.2 & 3,3 \\
\hline Yo1 & 24.2 & 5.7 & 2.6 & 15,15 \\
\hline Yi2 & 0.8 & 0.1 & 0.2 & 22,22 \\
\hline Yi6 & 4.0 & 1.1 & 0.5 & 17,17 \\
\hline Yi7 & 56.3 & $0.001^{*}$ & 1.2 & 0,0 \\
\hline Yo9 & 17.6 & 0.9 & 0.6 & 0,0 \\
\hline & $10^{-8}$ Torr & $10^{-8}$ Torr & $10^{-8}$ Torr & Meters
\end{tabular}

Table 4: Peak pressure rises of 4 unchanged Blue and 5 unchanged Yellow Q3-Q4 straight sections of Fill 7332 in Run5 and Fills 7554 and 7657 in Run6, and the NEG pipe installations. * The vacuum gauge at Yi7 pw3.2 was not logged at the time.

The vacuum gauge at Yi7 pw3.2 was not logged at the time of Fill 7554 and it was fixed shortly after that [2]. Later, for Fills 7640 and 7644, with 110-bunch mode and the total Yellow intensity of $125 \times 10^{11}$ and $118 \times$ $10^{11}$ protons, the Yi7 pressure rise were $0.55 \times 10^{-8}$, and $0.35 \times 10^{-8}$ Torr, respectively. These are consistent with the one shown in Table 4 for 7657 .

We note that there are significant pressure rise reduction at all 3 worst locations in Run5, i.e., Yo1, Yi7, and Yo9, with factors from 4 to more than 40. The concern of Yellow ring with larger electron cloud effect on beam in Run6 is somewhat relieved.

This pressure rise reduction is also presented in unchanged interaction regions, with perhaps smaller reduction ratio, shown in Table 5. 


\begin{tabular}{c|c|cc} 
Location & Peak Pressure & Peak Pressure & Peak Pressure \\
\hline & 7332 (Run5) & 7554 (Run6) & 7657 (Run6) \\
\hline \hline G1 & 7.9 & 2.4 & 0.57 \\
\hline G2 & 7.0 & 4.3 & 1.2 \\
\hline G5 & 0.10 & 0.08 & 0.04 \\
\hline G6 & 0.10 & 0.10 & 0.04 \\
\hline G7 & 4.5 & 2.4 & 0.65 \\
\hline G8 & 0.67 & 0.03 & 0.01 \\
\hline & $10^{-8}$ Torr & $10^{-8}$ Torr & $10^{-8}$ Torr
\end{tabular}

Table 5: Peak pressure rise at unchanged interaction regions IP2, IP6, and IP8 of Fill 7332 in Run5 and Fills 7554 and 7657 in Run6.

The fact that with no exception the pressure rises at all unchanged straight sections in Run6 is lower than that in Run5, in contrast with the observation in Run5, therefore, demands an explanation.

\section{Possible mechanism}

Among other changes, in Run6, the RHIC injection energy was lowered by $0.5 \mathrm{GeV}$, to avoid damaging resonance at AGS with de-polarization effects. The RHIC transition energy was lowered even further, therefore, the beam is injected into RHIC at relatively higher energy above the transition, compared with Run5. The RHIC transition $\gamma_{t r}$ and injection $\gamma_{i n j}$ are shown in Table 6 for Run5 (pp21) and Run6 (pp30).

\begin{tabular}{c|c|c|c||c} 
& RHIC & RHIC & Ratio & SPS \\
\hline & Run5 & Run6 & Run6/Run5 & \\
\hline \hline$\gamma_{t r}$ & 23.536 & 22.762 & & 22.8 \\
\hline$\gamma_{i n j}$ & 25.936 & 25.379 & & 28.72 \\
\hline$\gamma_{i n j}-\gamma_{t r}$ & 2.400 & 2.617 & 1.090 & 5.92 \\
\hline$|\eta|, 10^{-3}$ & 0.319 & 0.378 & 1.185 & 0.711
\end{tabular}

Table 6: RHIC transition and injection $\gamma$ in Run5 and Run6. CERN SPS parameters are also shown. 
The difference of RHIC injection at Run6 and Run5 is not large. Judged by $\gamma_{i n j}-\gamma_{t r}$, it is increased by $9 \%$. Nevertheless, judged by slippage factor it is increased by $18.5 \%$, perhaps not trivial.

It has been suspected that the RHIC electron cloud with long bunch spacing, 108 ns for 110-bunch and 216 ns for 55-bunch modes, might be assisted by positive ions generated from beam-chamber interaction [3, p.4]. The experiment of anti-grazing ridges seems to confirm that the beam-chamber interaction is indeed playing a role in electron multipacting [4]. Assuming the effectiveness of this mechanism, then the closer to the transition, the longer the secondary electron maybe survived in the bunch gap, and the stronger the electron multipacting.

As a comparison, the injection parameters of SPS [5] are also listed in Table 6, where the injection $\gamma_{i n j}$ is higher than transition $\gamma_{t r}$ by almost 6 units, more than double of that in RHIC.

The electron cloud observed at SPS is different from that in RHIC proton operations. The SPS electron cloud intensity threshold can be summarized in Table 7 [5],

\begin{tabular}{c|c|c|c|c} 
SPS EC Threshold & & & & \\
\hline \hline Bunch spacing, $n s$ & 25 & 50 & 75 & $130^{*}$ \\
\hline Bunch intensity, 10 11 & 0.3 & 0.6 & 1.2 & 2.5
\end{tabular}

Table 7: SPS electron cloud intensity threshold. * With the bunch spacing of $130 \mathrm{~ns}$, no electron cloud was observed for the bunch intensity of $2.5 \times 10^{11}$ protons.

At RHIC, the electron cloud was observed at the bunch spacings of 108 $n s$ and $216 n s$, with the bunch intensity higher than, say, $0.5 \times 10^{11}$ protons.

Despite the difference of the chamber size and conditions, the electron cloud induced pressure rise at SPS is somewhat uniform in the ring [6]. One electron detector can serve a purpose to estimate the electron cloud effect on the beam, which needless to say is determined by the electron cloud in the entire ring.

At RHIC, the electron cloud induced pressure rise depends on the locations. With same chamber size and conditions, local pressure rise could be different up to 4 orders of magnitude. Signal from one electron detector is, therefore, not representative for the entire ring. 
There are other differences of the electron cloud between RHIC and SPS, for example,

- Along with the energy ramp, the electron multipacting becomes stronger at SPS, presumably due to shorter and transversely smaller bunches. At RHIC, the strongest pressure rise is around beam injection. In early runs, the electron multipacting was decreased along the ramp.

- At RHIC, the electron cloud intensity threshold at long straight sections is much lower than that at the shorter ones. This is not observed at SPS.

The SPS electron cloud is consistent with the lifetime of secondary electron during the bunch gap, which was agreeable with the simulation and also measured on machines. Not surprisingly, therefore, the electron cloud observed at SPS is comparable with other machines. This is not the case for RHIC electron cloud [7].

\section{Possible studies}

The modification of the RHIC injection energy in terms of lowering the transition energy to improve longitudinal matching between AGS and RHIC in Run6 has proven successful. Since the pressure rise at injection is clearly reduces compared to previous runs, it would be desirable to experimentally test the effect of the difference between injection energy and transition energy on pressure rise at injection.

The easiest way in terms of RHIC optics modifications would be to revert the changes made to the lattice for Run6, namely to set the $\gamma_{t}$ quadrupoles to zero. This has the disadvantage that longitudinal matching between AGS and RHIC is difficult to achieve.

On the other hand, the RHIC transition energy can be lowered somewhat further using the same technique, though not very much. A transition value of $\gamma_{t}=22.40$ seems feasible, while even lower values cause tremendous optics distortions. With this setting, $\gamma_{i n j}-\gamma_{t r}$ is increased by $24 \%$ from Run5, and the slippage factor is increased by $38 \%$. 


\section{References}

[1] S.Y. Zhang, H.C. Hseuh, W. Fischer, H. Huang, and T. Roser, 'NEG Coating Application at RHIC', C-A/AP/220, BNL, Oct. 2005.

[2] R. Lee, private communication.

[3] S.Y. Zhang, 'RHIC Vaccum Pressure Bumps', C-A/AP/67, BNL, Jan. 2002.

[4] S.Y. Zhang, H.C. Hseuh, P. Thieberger, and D. Trbojevic, 'Effects of antigrazing ridges on electron cloud at the BNL Relativistic Heavy Ion Collider warm sections', Phys. Rev. ST Accel. Beams, 8, 123201, 2005.

[5] G. Arduini, in Proceedings of ELOUD04, p.31, Napa, CA, 2004, and in Proceedings of Two Stream Instability Workshop, Santa Fe, NM, 2001.

[6] J.M. Jimenez, ECLOU04, Napa, CA, April, 2004.

[7] F. Zimmermann, ECLOU04, Napa, CA, April, 2004. 